\title{
Contributions of phonological and verbal working memory to language development in adolescents with fragile $\mathrm{X}$ syndrome
}

\author{
Elizabeth I. Pierpont • Erica Kesin Richmond • \\ Leonard Abbeduto • Sara T. Kover • W. Ted Brown
}

Received: 13 March 2011 / Accepted: 27 September 2011 /Published online: 13 October 2011

(C) Springer Science+Business Media, LLC 2011

\begin{abstract}
Fragile X syndrome (FXS) is the most common inherited cause of intellectual disability. Although language delays are frequently observed in FXS, neither the longitudinal course of language development nor its cognitive predictors are well understood. The present study investigated whether phonological and working memory skills are predictive of growth in vocabulary and syntax in individuals with FXS during adolescence. Forty-four individuals with FXS (mean age $=12.61$ years) completed assessments of phonological memory (nonword repetition and forward digit recall), verbal working memory (backward digit recall), vocabulary, syntax, and nonverbal cognition. Vocabulary and syntax skills were reassessed at a 2-year follow-up. In a series of analyses that controlled for nonverbal cognitive ability and severity of autism symptoms, the relative contributions of phonological and working memory to language change over time were investigated. These relationships were examined separately for boys and girls. In boys with FXS, phonological memory
\end{abstract}

\author{
E. I. Pierpont $(\square)$ \\ Department of Psychology, University of Wisconsin-Madison, \\ 1202 W. Johnson Street, \\ Madison, WI 53706, USA \\ e-mail: eipierpont@wisc.edu \\ W. T. Brown \\ Institute for Basic Research in Developmental Disabilities, \\ Staten Island, NY, USA \\ E. K. Richmond $\cdot$ S. T. Kover \\ Waisman Center, University of Wisconsin-Madison, \\ 1500 Highland Avenue, \\ Madison, WI 53705, USA \\ L. Abbeduto \\ UC Davis MIND Institute, University of California-Davis, \\ 2825 50th Street, \\ Sacramento, CA 95817, USA
}

significantly predicted gains in vocabulary and syntax skills. Further, verbal working memory was uniquely associated with vocabulary gains among boys. In girls with FXS, phonological and working memory skills showed no relationship with language change across the 2-year time period. Our findings indicate that, for adolescent boys with FXS, acquisition of vocabulary and syntax may be constrained by the ability to maintain and manipulate phonological representations online. Implications for the identification and treatment of language disorders in this population are discussed. The present study is the first to identify specific cognitive mechanisms contributing to language growth over time in individuals with FXS.

Keywords Fragile X syndrome $\cdot$ Language $\cdot$ Phonological memory $\cdot$ Working memory $\cdot$ Nonword repetition · Digit span

\section{Introduction}

Fragile X syndrome (FXS), a genetic disorder resulting from a mutation of the FMR1 gene, is the most common inherited cause of intellectual disability (Mazzocco 2000). Recent epidemiological studies report a prevalence of approximately 1 in 2,500 individuals for the full mutation (FernandezCarvajal et al. 2009; Hagerman 2008; Pesso et al. 2000). In FXS, the mutation results in a reduction or absence of FMRP, the protein produced by the FMR1 gene. This reduction in FMRP levels is thought to account for a range of physical, behavioral, and neuropsychological features and deficits observed in affected individuals (Loesch et al. 2004). Males with FXS typically exhibit a more severe phenotype than females, characterized by moderate to severe intellectual disability (Loesch et al. 2004; Skinner et al. 2005). Among females, cognitive functioning ranges from within the average 
range to moderate intellectual disability (Cronister et al. 1991; de Vries et al. 1996). FXS also affects social development, and behaviors characteristic of autism are frequently observed. The percentage of individuals with FXS meeting the diagnostic criteria for autism has been estimated at $25-50 \%$, with virtually all males with FXS displaying at least some behaviors characteristic of autism (Philofsky et al. 2004; Demark et al. 2003; Lewis et al. 2006; Kaufmann et al. 2004).

In addition to cognitive and social impairments, substantial delays in language are characteristic of the FXS phenotype (Sudhalter et al. 1991; Murphy and Abbeduto 2003). Individuals with FXS demonstrate delays in receptive and expressive language that span multiple domains. Impairments may be evident in vocabulary size and complexity, morphosyntax (knowledge of the formation and structure of words), and syntax (application of principles for constructing sentences; see Finestack et al. 2009). Deficits in social aspects of language (e.g., maintenance of a conversation topic, appropriate turn-taking) and frequent use of repetitive language are also common (Belser and Sudhalter 2001; Murphy and Abbeduto 2007; Roberts et al. 2007; Mazzocco et al. 2006). Although considerable research has documented a characteristic profile of difficulties with acquisition and use of language among groups of individuals with FXS relative to their chronological-age peers, it is also important to acknowledge the high degree of within-syndrome variability in language outcomes (Abbeduto et al. 2007). For example, children and adolescents with FXS exhibit striking variation in expressive skills, with some individuals limited to nonverbal communication and others (mostly females) exhibiting age-appropriate competence with most language mechanics (Murphy and Abbeduto 2003; Mazzocco et al. 2006).

It has been speculated that differences among individuals in language functioning arise from a combination of biological influences, neurocognitive and behavioral characteristics, and variations in the linguistic and social environment (Finestack et al. 2009). Identifying key predictors of linguistic outcomes in FXS may be useful for a number of reasons. First, given the prevalence of language impairments in this population and the profound impact communication deficits can have on quality of life and educational attainment (e.g., Van Agt et al. 2011; Durkin et al. 2009), developing a better understanding of sources of language variation in FXS is of considerable public health importance. Such knowledge could aid in identifying early markers of language impairment and in counseling families regarding prognosis and treatment recommendations. Second, knowledge of the underlying factors that predict language outcomes could be beneficial for constructing more effective interventions for language problems in affected individuals. Finally, a better understanding of the longitudinal course and influences on language development in this population may have relevance not only for individuals with FXS, but also for the identification and treatment of language difficulties within the general population and other neurodevelopmental disorders. Unfortunately, there have been few attempts to empirically test the factors that shape differences in language among individuals with FXS.

Although the sources of variation in language functioning in FXS are not fully understood, two factors - gender and autism status - are known to contribute to that variation. Specifically, male gender and comorbid autism diagnosis have been shown to place an individual with FXS at significantly higher risk for language impairments (Philofsky et al. 2004; Finestack et al. 2009; Abbeduto et al. 2003). What remains unclear is the extent to which differences between these groups (e.g., females vs. males with FXS; FXS-only vs. FXS with autism) in language outcomes at a given time point actually reflect differences in other aspects of neuropsychological functioning, such as general intellectual ability or specific cognitive or socioemotional functions. Thus, whereas a number of studies have succeeded in identifying FXS subgroups that differ in language ability, a mechanistic explanation for these differences is still lacking. Further, little is known about how specific neurocognitive functions relate to growth over time in specific language skills, such as vocabulary and syntax.

In order to begin to disentangle key mechanisms that lead to variation in language acquisition and use in FXS, a first step is to develop a better understanding of how impairments in specific areas of cognition play a role in the development of language in this population. Moreover, it is reasonable to focus on those factors that have been demonstrated to account for individual differences in the typically developing population, or those known to discriminate individuals with language impairment from those without. One such cognitive skill is working memory. Working memory refers to the short-term storage and manipulation of information necessary for a range of higher cognitive functions (Baddeley 2003; Smith and Jonides 1999). In many cognitive models, working memory is divided into several distinct processes or subdomains. Commonly, visuospatial working memory is posited to be separate from verbal working memory (e.g., Baddeley 1986; Logie 1995). This distinction is largely supported by neuroimaging evidence, as tasks involving these types of information activate different hemispheres (D'Esposito et al. 1998; Smith et al. 1996) and cortical regions (Lycke et al. 2008; Walter et al. 2003).

With respect to developmental language disorders, the role of verbal memory in particular has engendered a great deal of research. In some models of working memory, such as that of Baddeley (1986), the temporary storage and rehearsal of phonological representations is thought to occur within a distinct cognitive system (often referred to as the "phonological loop"), which is hypothesized to be critical for learning the novel phonological forms of new words 
(Baddeley et al. 1998). Other theorists have argued that working memory does not constitute a separate dedicated cognitive system, but rather reflects chiefly the activation of knowledge or attentional focus on those representations that are involved in language comprehension and production (Cowan 1997; MacDonald and Christianson 2002; Acheson and MacDonald 2009). In acknowledgment of the merits of different competing theories, we will use the more theoretically neutral term "phonological memory" to refer to the process of maintenance and rehearsal of phonological representations. This process of verbatim recall of verbal information has also been referred to as "verbal short-term memory" by some researchers (e.g., Brock and Jarrold 2004; Alloway et al. 2006). Tasks thought to recruit this mechanism include tests of nonword repetition, digit span, and word span. These types of phonological memory tasks typically result in the activation of a brain network that includes the left inferior frontal and premotor cortex, supplementary motor areas, and cerebellum (Lycke et al. 2008; Paulesu et al. 1993; Awh et al. 1996).

Studies of typically developing children have revealed robust associations between performance on phonological memory tasks and word learning during preschool years through about the age of 8 years (Gathercole et al. 1992; Bowey 2001). Additionally, considerable research has also demonstrated that children with specific language impairment perform more poorly than their peers on phonological memory tasks, especially nonword repetition (e.g., Gathercole and Baddeley 1990; Ellis Weismer et al. 2000; Graf Estes et al. 2007). Thus, it has been hypothesized that impaired phonological memory may constitute an important cognitive risk factor for language impairments (Gathercole and Baddeley 1990).

Research on other genetic syndromes associated with atypical language development has also lent support to this notion. In individuals with Down syndrome and Williams syndrome, strong associations between nonword repetition performance and measures of receptive vocabulary and grammar have been reported; moreover, the magnitude of these relationships is consistently stronger in these groups than for mental age- or language-matched controls (Laws 1998; Chapman 2006; Cairns and Jarrold 2005; Grant et al. 1997; Robinson et al. 2003). In a longitudinal study of adolescents with Down syndrome, Laws and Gunn (2004) demonstrated that phonological memory skills were a significant predictor of vocabulary development over a 5-year span. Among younger participants (ages 10-14), performance on these tasks was also predictive of the acquisition of syntax. Thus, phonological memory ability may be closely related not only to concurrently measured language levels, but also to the rate of language growth over time in individuals with intellectual disabilities.

In the present study, we examined the contribution of phonological memory to the rate of growth in language in individuals with FXS. Although some research indicates that phonological memory skills are impaired in individuals with FXS compared to control groups with and without developmental delays (Munir et al. 2000; Cornish et al. 2009; Baker et al. 2011), the question of whether phonological memory skills constitute a specific deficit relative to other domains of memory has not been conclusively resolved. A recent study suggests that young boys with FXS not only exhibit impairments in phonological memory in comparison to typically developing mental age-matched controls, but also more severe difficulties on phonological memory tasks in comparison with visual-spatial memory tasks (Baker et al. 2011). Thus, we hypothesized that these observed impairments in phonological memory might have a particularly negative impact on language growth in FXS or perhaps signal a vulnerability in brain circuitry important for language. However, given other important aspects of the FXS phenotype such as low IQ, higher rates of autism symptomatology, and marked gender differences, it is also plausible that phonological memory deficits could play a less pivotal role in language development for individuals with FXS than for other populations. The current study aimed to distinguish the relative contributions of these different factors to language growth in FXS.

Although we have argued that investigating the role of cognitive factors (and phonological memory in particular) in language development in FXS has significant scientific and clinical implications, additional aspects of working memory should also be considered when studying FXS. Some evidence suggests that, across a variety of short-term memory tasks, performance of individuals with FXS depends less on the type of processing than on the degree of cognitive control required. Indeed, boys with FXS appear to have particular difficulty with tasks demanding high levels of attention or cognitive processing in order to manipulate information maintained in memory, compared with other types of shortterm memory tasks (Lanfranchi et al. 2009; Cornish et al. 2001). Further, individuals with FXS show marked deficits on tasks of executive control, attention, and inhibition relative to individuals with comparable degree of developmental delay (Hooper et al. 2008). Therefore, it is important to also consider the role of executive processes in the development of language skills in this population.

In many working memory models, executive processes necessary for active planning, manipulation, or organization of items held in memory are considered to be separate from storage capacity (Smith et al. 1996; D'Esposito 1999). In Baddeley's (2003) model, this process corresponds to the "central executive." This type of online processing can be measured in the verbal domain using tasks such as the backward digit span and letter-number sequencing. Although there are various terms that have been used to describe this process, we will henceforth refer to this simply as "verbal working memory." This term emphasizes both the need for verbal maintenance as well as active processing/manipulation 
and has been used in other studies investigating these cognitive processes (Archibald and Gathercole 2006; Conklin et al. 2000). Neuroimaging studies suggest that verbal working memory tasks activate areas of the brain that are not active during simple phonological storage or rehearsal tasks, including anterior cingulate and dorsolateral prefrontal cortex (Smith and Jonides 1999; Wager and Smith 2003). Some research has revealed that, in addition to the welldocumented deficits in phonological memory, children with language impairments also have significant impairments in verbal working memory tasks (Archibald and Gathercole 2006). This finding raises the possibility that a combination of cognitive deficits, including in phonological and verbal working memory, may be responsible for difficulties in language learning for many individuals.

In summary, research on language development in typically developing children and those with language impairments suggests that phonological and verbal working memory skills have the potential to explain significant variation in language outcomes. In the present study, we investigated the contributions of phonological and verbal working memory to language outcomes in individuals with FXS over a 2-year period. The study was designed to address the following questions:

1. To what extent do phonological and verbal working memory skills predict the development of vocabulary and syntax skills among individuals with FXS during adolescence, after controlling for nonverbal intellectual ability and autism symptom severity?

2. Do these relationships vary by gender?

\section{Methods}

\section{Participants}

Participants were 44 adolescents with FXS (30 boys and 14 girls) who were enrolled in a longitudinal study of language and cognitive development. Participants ranged in age from 10 to 16 years at the time of the initial session $(M=12.61, \mathrm{SD}=1.77)$. The current sample includes all individuals in the longitudinal study who completed the language measures at both the initial session (time 1) and a follow-up session 2 years later (time 2). The sample contained five sets of sibling pairs. All participants were native English speakers who passed a hearing screening indicating a pure-tone threshold of $<30 \mathrm{~dB}$ in at least one ear. The sample was largely middle class, with median family income in the $\$ 50,000-60,000$ range. Fortyeight percent of participants' mothers had received a college degree or higher. Regarding race/ethnicity, $93 \%$ of families identified the participating youth with FXS as Caucasian, 5\% as African American, and 2\% as Native American.
Families were recruited for the study through newspaper advertisements, nationwide radio announcements, a university registry of families with children who have developmental disabilities, and postings on internet sites, listservs, and newsletters of developmental disability organizations. The study was approved by the Institutional Review Board at the University of Wisconsin-Madison. Parents provided written informed consent prior to participation. Molecular confirmation of an FMR1 full mutation based on analysis of a peripheral blood sample was obtained for all participants. Additionally, all participants were evaluated for autism symptoms using the Autism Diagnostic Observation Schedule (ADOS; Lord et al. 1999) and the Autism Diagnostic Interview, Revised (ADI-R; Lord et al. 1994), each administered by a research-reliable examiner. Eleven participants ( $25 \%$; 10 boys and 1 girl), met the criteria for autism on both of these instruments, a rate consistent with other reported prevalence estimates for this population (Philofsky et al. 2004; Demark et al. 2003; Lewis et al. 2006; Kaufmann et al. 2004). Note that the current sample of individuals with FXS is partially overlapping with the participant sample from another published study (McDuffie et al. 2010); however, different measures and research questions were the focus of each paper.

\section{Procedures}

All behavioral tests were administered by well-trained graduate-level research assistants with extensive experience working with individuals with developmental disabilities, including those with FXS. At each annual assessment, participants completed a battery of language and cognitive assessments that was administered in two to three sessions over the course of 2 days. In most cases, all measures reported here were administered to a participant by the same examiner at any given time point. Previsit interviews with parents solicited information about useful reinforcers, effective styles of interaction, and signs of anxiety or distress for their sons and daughters, information which was then used to maximize participant comfort and compliance. Photos and names of examiners were shared with families in advance of testing to decrease participant anxiety. Participants were also given an opportunity to acclimate to the testing situation prior to attempting any of the tasks and visual schedules were used to help decrease anxiety and increase attentiveness. In addition, examiners were trained to monitor participant behavior so as to appropriately use reinforcers and breaks from testing to ensure participants performed to the best of their ability.

\section{Measures}

Phonological memory At time 1, participants were administered the Nonword Repetition Task (NRT) developed by 
Dollaghan and Campbell (1998). The stimulus set for this test consists of four nonwords at each of four-syllable lengths (one, two, three, and four syllables). Test items from the NRT were administered through an RCA CD player. Participant responses were audiorecorded by an external microphone onto a cassette recorder (Marantz PMD 201) for phonetic transcription. The entire protocol for each participant was transcribed and scored independently by a trained research assistant who was not one of the examiners. Audiotaped responses from $18 \%$ of the sample were independently transcribed and scored by a second trained research assistant. The following phoneme-by-phoneme percentages of interscorer agreement were obtained using Cohen's kappa (Cohen 1960): one-syllable words $=0.92$; two-syllable words $=0.90$; three-syllable words $=0.92$; foursyllable words $=0.93$; total correct $=0.95$.

In order to avoid inclusion of NRT scores that were based on only a small number of items, participants who did not attempt to repeat more than half of the 16 items administered in the task $(n=5)$ were excluded from any analysis that used this measure, but were included in all other analyses. These five participants were clear outliers with respect to responding, with number of items attempted at $>1.5 \mathrm{SD}$ below the group average. For one additional participant, the NRT task was not administered due to behavioral difficulties during the session. For those who completed the task according to the above criteria $(n=38)$, scores indexing the percentage of phonemes correct (PPC) were calculated based on the number of phonemes correctly repeated, divided by the number of phonemes attempted, at each syllable length (one to four syllables). Items for which there was no response were thus factored out of the PPC score, as it was not possible to ascertain whether the response would have been correct or incorrect if the item had been attempted. Additionally, a total PPC summary score across the entire set of nonwords was calculated for each participant.

In addition to the NRT task, the digit recall subtest from the Working Memory Test Battery for Children (WMTB-C; Pickering and Gathercole 2001) was also administered to measure phonological memory at time 1 . In this test, a series of numbers is spoken by the examiner and participants are asked to recall the list in the same order in which it was spoken. Digit lists begin with one item, and sequence length is increased by one digit if the participant correctly recalls four (out of six) sequences at a given length, up to a maximum of nine digits. The test is discontinued after three or more errors are made at a given sequence length. A total digit recall score is calculated based on the number of digit lists correctly recalled across the test. Data were missing for one participant on this task due to an examiner administration error.

Verbal working memory To assess verbal working memory, the backward digit recall subtest from the WMTB-C was administered. This test has the same structure as the digit recall test, except that participants are asked to recall lists of digits in the reverse order of the sequence spoken to them. Thus, this test requires additional processing to manipulate the sequence order. Because many individuals with developmental disabilities have difficulty understanding the instruction to "say it backwards," all participants received explicit training, with visual supports, to illustrate the steps involved in the task. This training helped to ensure understanding of the task prior to test item administration. A total backward digit recall score was calculated based on the number of correct responses. Data were missing for two participants: one participant who failed to attempt any trials of this task and another for whom the task was not administered due to behavior problems.

Standardized language measures The following language measures were administered at both time 1 and time 2. Each measure yields a raw score (based on the number of items or blocks passed), a standardized score (based on performance relative to a norming sample), and an age-equivalent score (based on the median chronological age at which a raw score was obtained within the norming sample). Receptive vocabulary was measured using the Peabody Picture Vocabulary Test, Third Edition (PPVT-III; Dunn and Dunn 1997). In this task, the examinee is asked to select a picture from a fourpicture array that best matches a word spoken by the examiner. The Test for Reception of Grammar, Second Edition (TROG-2; Bishop 2003) was administered to evaluate receptive syntax. The TROG-2 assesses comprehension of sentences of varying syntactic complexity, reflecting differences in word order, function words, and grammatical inflections. Participants are asked to select one picture out of an array of four that matches a sentence spoken by the examiner. Items are presented in blocks of four, with all the items in the block exemplifying the same syntactic construct (e.g., the plural morpheme). A block is considered to be passed only if all four items are answered correctly.

Expressive language was assessed using the Expressive Vocabulary Test (EVT; Williams 1997) and the Syntax Construction Test from the Comprehensive Assessment of Spoken Language (CASL; Carrow-Woolfolk 1999). In the EVT, expressive vocabulary knowledge is elicited using pictures and examiner prompts. The CASL Syntax Construction (CASL-SC) subtest measures a participant's ability to generate sentences using a variety of targeted morphosyntactic rules. Sentences are elicited by asking the examinee to formulate a word, phrase, or sentence that is semantically and grammatically compatible with a verbal stimulus and a picture.

Nonverbal cognition Due to the expected contribution of intellectual functioning in predicting language development, nonverbal cognitive ability was measured at time 1 
using the Leiter International Performance Scale-Revised (Leiter-R; Roid and Miller 1997) Brief IQ Screener. The Brief IQ index is based on four subtests that assess visualization (figure ground and form completion) and fluid reasoning (sequential order and repeated patterns). The Leiter- $\mathrm{R}$ has strong psychometric properties and is widely used in research on individuals with developmental disabilities, including FXS (e.g., Hooper et al. 2000; Kuschner et al. 2007). Instructions are pantomimed and responses from the participant are nonverbal (e.g., placing cards in a row). This measure yields a standardized nonverbal Brief IQ score based on age norms. In addition, a growth score can be derived from the Leiter-R based on the participant's raw score. Growth scores are thought to reflect the examinee's absolute level of ability at a given time point. Although conceptually similar to mental ages, growth scores, unlike mental ages, have equal-interval measurement properties and are applicable for an ability range from toddlerhood to adulthood. Thus, growth scores are particularly useful in examining populations with significant cognitive delays (Hooper et al. 2000). The growth score metric was used as the primary measure of nonverbal intellectual functioning for statistical analyses in the current study.

Autism symptom severity Individuals with FXS who have a comorbid diagnosis of autism have been shown to differ from those without autism in aspects of language skill, such as receptive language ability and intelligibility, although some research suggests that broad expressive language profiles may be similar in these groups (Lewis et al. 2006; Kover and Abbeduto 2010). Although the description of autism in FXS was not a focus of the current study (for further exploration of this issue, see McDuffie et al. 2010; McDuffie et al., in press), previous investigations of FXS suggest that this may be an important aspect of the phenotype to consider in language research. For the purposes of the current study, autism symptoms were quantified by calculating a severity score based on each participants' scores on the ADOS. Autism severity scores were derived using an algorithm developed by Gotham et al. (2009). This algorithm can be applied across three different ADOS modules and yields a severity score between 1 and 10. A continuous measurement of autism severity was deemed preferable to using a categorical distinction (e.g., autism vs. no autism) for several reasons. First, some research suggests that the diagnostic results of gold-standard measures such as the ADOS and ADI-R do not always converge in FXS (Harris et al. 2008). Second, social and communication skills may differ significantly in individuals with FXS and autism from those with idiopathic autism, so a categorical distinction between these groups may be problematic in this population (Hall et al. 2010).
Finally, some recent research suggests that a continuous measure of autism severity is more sensitive to relationships with language measures and avoids the problem of some individuals falling into an unclear diagnostic category (McDuffie et al., in press).

In the current sample, autism severity scores covered the full range from 1 to $10(M=4.88, \mathrm{SD}=3.15)$. Severity scores were obtained using the standard method described by Gotham et al. (2009) for all but three participants. For two female participants, a severity score could not be obtained because these individuals were administered module 4 of the ADOS based on their developmental level and the severity algorithm applies only to modules $1-3$. These two scores were treated as missing data. For a third participant, a male with FXS who had just passed the age range containing norms for module 1 (age at test administration $=15$ years, 2 months), a severity score was derived based on module 1 norms for the 6- to 14-year-old age group.

\section{Results}

Descriptive data and concurrent comparisons

Table 1 presents descriptive statistics for the participant group at both time points, including age, nonverbal IQ, and ageequivalent scores on each of the language measures. As expected, girls scored consistently higher than boys across all of the cognitive and language measures, despite no significant difference in chronological age between the genders. Note that the TROG-2 task was especially difficult for many boys with FXS compared with other measures, and several participants scored at or near the age-equivalent floor for this measure. However, a wide variation in raw scores was observed for all language measures in both boys and girls. At time 1, raw scores for all four language measures were strongly correlated with Leiter-R growth scores (PPVT-III, 0.77; EVT, 0.81; TROG-2, 0.84; CASL-SC, $0.79, p<0.001$ for all correlations), indicating that variation in language at a given time point is closely tied to intellectual ability.

Regarding phonological memory, Fig. 1 presents the PPC on the NRT for each syllable length, grouped according to gender. A mixed-model, repeated-measures ANOVA with Greenhouse-Geisser correction was conducted to test for gender differences at each syllable length on the NRT. The analysis revealed a significant main effect of syllable length, $F(2.35,84.43)=72.43, p<0.001$, partial $\eta^{2}=0.668$, and a significant gender effect, $F(1,36)=9.75, p<0.01$, partial $\eta^{2}=$ 0.213 , with girls outperforming boys on this task. The syllable length $\times$ gender interaction was not significant. In order to examine whether the observed gender differences in NRT performance were driven by differences in nonverbal cogni- 
Table 1 Descriptive statistics for age, IQ, and language measures at time 1 and time 2, grouped according to gender

\begin{tabular}{|c|c|c|c|c|c|c|c|c|c|}
\hline \multirow[t]{2}{*}{ Measure } & & \multicolumn{4}{|c|}{ Time 1} & \multicolumn{4}{|c|}{ Time 2} \\
\hline & & $n$ & Mean & $\mathrm{SD}$ & Range & $n$ & Mean & SD & Range \\
\hline \multirow[t]{2}{*}{ Chronological age } & Girls & 14 & 12.04 & 1.57 & $10.18-15.59$ & 14 & 14.02 & 1.53 & $12.18-17.45$ \\
\hline & Boys & 30 & 12.87 & 1.81 & $10.17-16.01$ & 30 & 14.88 & 1.81 & $12.21-18.00$ \\
\hline \multicolumn{10}{|l|}{ Nonverbal cognition } \\
\hline \multirow[t]{2}{*}{ Leiter-R brief IQ } & Girls & 14 & 66.86 & 13.96 & $46-91$ & 14 & 66.00 & 18.76 & $38-95$ \\
\hline & Boys & 30 & 46.03 & 8.64 & $36-65$ & 30 & 42.97 & 8.84 & $36-67$ \\
\hline \multicolumn{10}{|l|}{ Vocabulary } \\
\hline \multirow[t]{2}{*}{ PPVT-III age equivalent } & Girls & 14 & 10.10 & 2.58 & $5.3-14.8$ & 14 & 11.04 & 2.91 & $5.67-17.00$ \\
\hline & Boys & 30 & 6.55 & 2.47 & $2.4-14.3$ & 30 & 6.99 & 3.04 & $1.75-17.42$ \\
\hline \multirow[t]{2}{*}{ EVT age equivalent } & Girls & 14 & 9.00 & 2.43 & $5.42-13.83$ & 14 & 10.44 & 2.84 & $6.33-17.33$ \\
\hline & Boys & 30 & 5.43 & 1.97 & $3.17-10.92$ & 29 & 6.12 & 2.52 & $3.58-13.58$ \\
\hline \multicolumn{10}{|l|}{ Syntax } \\
\hline \multirow[t]{2}{*}{ TROG-2 age equivalent } & Girls & 14 & 7.06 & 3.04 & $<4.00-12.00$ & 14 & 8.03 & 2.90 & $4.42-12.00$ \\
\hline & Boys & 30 & 4.43 & 0.94 & $<4.00-7.92$ & 30 & 4.66 & 1.58 & $<4.00-10.83$ \\
\hline \multirow[t]{2}{*}{ CASL-SC age equivalent } & Girls & 14 & 7.81 & 2.43 & $2.17-11.33$ & 14 & 9.42 & 3.12 & $3.25-14.25$ \\
\hline & Boys & 29 & 4.17 & 2.06 & $2.08-9.67$ & 28 & 4.40 & 2.37 & $2.08-10.00$ \\
\hline
\end{tabular}

PPVT-III Peabody Picture Vocabulary Test, Third Edition, EVT Expressive Vocabulary Test, TROG-2 Test for Reception of Grammar, Second Edition, CASL-SC Comprehensive Assessment of Spoken Language, Syntax Construction subtest

tive ability between boys and girls, Leiter-R growth scores were added to the model as a covariate. In this model, nonverbal cognitive skills accounted for significant variance in nonword repetition skills, $F(1,35)=5.18, p<0.05$, partial $\eta^{2}=0.129$, and the gender effect was no longer significant.

On the WMTB-C digit recall task, the number of digit lists correctly recalled by any given participant ranged from 6 to 31. In a one-way ANOVA, girls scored significantly higher than boys on the digit recall task, $F(1,41)=23.92, p<0.001$, partial $\eta^{2}=0.368$. When added as a covariate to this analysis,

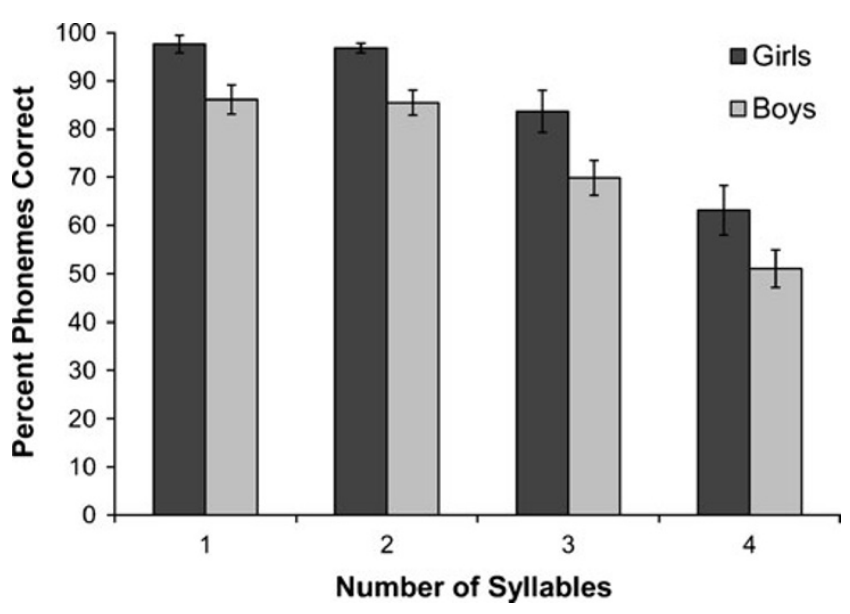

Fig. 1 Average percent phonemes correct at four different syllable lengths on the NRT for 38 individuals with FXS. Error bars represent 1 standard error
Leiter-R growth scores explained significant variance in digit recall scores, $F(1,40)=16.73, p<0.001$, partial $\eta^{2}=0.295$; however, the gender difference in digit recall scores remained significant after adding the covariate, $F(1,40)=$ 5.54, $p<0.05$, partial $\eta^{2}=0.122$. Thus, gender differences on this measure were not fully accounted for by differences in intellectual functioning.

For the WMTB-C backward digit recall task, the number of digit lists that participants were able to correctly recall (in reverse order) ranged from 0 to 19 . Girls again scored significantly higher than boys, $F(1,40)=26.84, p<0.001$, partial $\eta^{2}=0.402$. When added as a covariate to this analysis, Leiter-R growth scores explained significant variance in backward digit recall scores, $F(1,39)=22.02, p<0.001$, partial $\eta^{2}=0.361$, but the gender difference in backward digit recall scores remained significant after adding the covariate, $F(1,39)=6.05, p<0.05$, partial $\eta^{2}=0.134$.

Language change in adolescents with FXS

Improvements in language abilities were observed over the 2-year span for most of the language measures. Changes in raw scores were generally more pronounced for girls than for boys (see Table 2). For example, on the EVT, the girls improved on average by 10.93 raw score points from time 1 to time 2 (i.e., they correctly produced approximately 11 additional vocabulary items at the follow-up testing than they had at the initial session), whereas the boys improved on average by 5.14 raw score points (i.e., fewer than 6 
Table 2 Difference scores indicating mean change in raw scores for language assessments over a 2-year time period in individuals with FXS, separated according to gender

\begin{tabular}{|c|c|c|c|c|c|c|}
\hline & & \multirow[t]{2}{*}{ Number } & \multicolumn{2}{|c|}{$\begin{array}{l}\text { Raw score change } \\
\text { (time } 2 \text {-time } 1 \text { ) }\end{array}$} & \multicolumn{2}{|c|}{$\begin{array}{l}\text { One-sample } \\
t \text { test }\end{array}$} \\
\hline & & & Mean & $\mathrm{SD}$ & $t$ & $p$ \\
\hline \multirow[t]{2}{*}{ PPVT-III } & Girls & 14 & 7.07 & 6.82 & 3.88 & $<0.01$ \\
\hline & Boys & 30 & 4.00 & 11.63 & 1.88 & $<0.05$ \\
\hline \multirow[t]{2}{*}{ EVT } & Girls & 14 & 10.93 & 6.99 & 5.85 & $<0.001$ \\
\hline & Boys & 29 & 5.14 & 7.98 & 3.47 & $<0.01$ \\
\hline \multirow[t]{2}{*}{ TROG-2 } & Girls & 14 & 2.29 & 2.16 & 3.95 & $<0.01$ \\
\hline & Boys & 30 & 0.43 & 2.46 & 0.965 & n.s. \\
\hline \multirow[t]{2}{*}{ CASL-SC } & Girls & 14 & 5.21 & 3.62 & 5.39 & $<0.001$ \\
\hline & Boys & 27 & 1.89 & 5.35 & 1.83 & $<0.05$ \\
\hline
\end{tabular}

n.s. not significant

additional items). This suggests that the girls not only started out higher than the boys in language ability, but they also developed their language skills at a faster rate during the study period. For one measure, the TROG-2, boys did not demonstrate significant improvement over the 2-year period.

Relationship between phonological and verbal working memory skills and language change

We next investigated whether the phonological and working memory measures at time 1 were predictive of progress in language skills over the 2-year study period. Using partial correlations, the relationship between these measures at time 1 and scores on the vocabulary and syntax tests at time 2 was examined, controlling for Leiter-R growth scores, autism symptom severity, and the corresponding time 1 language test scores. For all language and memory tests, raw scores at the designated time point were utilized. Use of raw scores was deemed to be methodologically preferable to the use of standard scores, which tend to suffer from large floor effects in populations with developmental disabilities (Hessl et al. 2009; Mervis 2004). Raw scores were also considered preferable to age-equivalent scores, which are measured on an ordinal rather than interval scale and thus have less desirable measurement characteristics for the current statistical techniques (Mervis and Klein-Tasman 2004).

Analyses were conducted separately for each of the predictors. In addition, because of the marked differences in language abilities in girls and boys with FXS, separate analyses were conducted for each gender. In order to correct for multiple tests, the Holm step-down correction was applied to control significance levels (Holm 1979). This correction was applied separately to vocabulary and syntax tests, which measure theoretically distinct con- structs. Because our hypothesis was directional (e.g., that stronger phonological memory skills would be predictive of greater improvements over time in language skills), onetailed tests were performed.

Results of the partial correlation analyses are presented in Table 3. For boys with FXS, nonword repetition was significantly correlated with receptive and expressive vocabulary and expressive syntax at time 2 , when controlling for those measures at time 1 as well as autism symptom severity and nonverbal cognition. Similarly, significant correlations were seen between digit recall and time 2 receptive and expressive vocabulary. In addition, working memory (backward digit recall) was a significant correlate of boys' outcomes in receptive vocabulary and receptive syntax.

In contrast to the pattern seen among boys with FXS, phonological and verbal working memory tests were not significantly associated with any language outcomes in girls with FXS. In some cases, correlations were not even in the expected (positive) direction (see Table 3). In order to ensure that these results were not related to undue influence by the covariates in these relationships, partial correlations were also examined without the covariates. All partial correlations between language measures and phonological and working memory measures, controlling for time 1 language skills, remained nonsignificant (results without covariates not reported here, but are available from the authors). Thus, although correlations between time 1 and time 2 test results for each measure were very robust in this group ( $p<0.001$ for all measures using Pearson correlations), suggesting strong reliability of each measured

Table 3 Partial correlations between phonological and working memory skills at time 1 and language measures at time 2, controlling for corresponding time 1 measures, autism symptom severity, and nonverbal cognitive ability

\begin{tabular}{lcclll}
\hline \multirow{2}{*}{ Time 1 } & \multicolumn{3}{l}{ Time 2} & & \\
\cline { 2 - 3 } & \multicolumn{3}{l}{ Vocabulary } & & \multicolumn{2}{l}{ Syntax } & \\
\cline { 2 - 3 } \cline { 5 - 6 } & PPVT-III & EVT & & TROG-2 & CASL-SC \\
\hline Boys & & & & \\
Nonword repetition & $0.54^{*}$ & $0.37^{*}$ & 0.24 & $0.48^{*}$ \\
Digit recall & 0.36 & 0.39 & & $0.42^{*}$ & $0.54^{*}$ \\
Backward digit recall & $0.44^{*}$ & 0.28 & & $0.62^{*}$ & 0.33 \\
Girls & & & & \\
Nonword repetition & 0.43 & -0.64 & & 0.11 & -0.04 \\
Digit recall & 0.22 & -0.15 & -0.59 & 0.32 \\
Backward digit recall & -0.25 & -0.45 & -0.60 & 0.43 \\
\hline
\end{tabular}

Partial correlations were calculated using raw scores for language and memory measures and Leiter-R growth scores for nonverbal cognition ${ }^{*} p<0.05$, statistically significant, one-tailed, with Holm correction 
language construct, phonological and working memory did not significantly predict language outcomes over time among girls.

In order to investigate the relative contributions of phonological memory and verbal working memory to language change over time, a set of two regression analyses was conducted. In each regression model, Leiter-R growth scores and autism symptom severity were included as control variables. These analyses only included boys with FXS because the previous correlational analyses revealed no consistent relationship between phonological and working memory predictors and language growth among girls. The first analysis focused on vocabulary growth. In order to create an index of vocabulary development over time, change scores were calculated for each participant based on the difference in raw scores from time 1 to time 2 on the PPVT-III and EVT. A vocabulary change composite score was obtained by adding the standardized change scores ( $z$ scores) for both vocabulary measures. For one participant who did not have available EVT scores at both time points, only the PPVT-III change score was used. A multiple regression analysis was conducted to examine contributions to this vocabulary change composite, with time 1 nonverbal cognition (Leiter-R growth scores), autism symptomatology (autism severity score), phonological memory (PPC on the NRT task), and verbal working memory (backward digit recall) entered as predictors. Note that NRT performance was used as the phonological memory variable; however, results using the digit recall measure revealed similar results (and are available from the authors). Overall, results indicated that these predictors accounted for $68 \%$ of the variance in vocabulary growth, $F$ $(4,23)=9.95, p<0.001$. Both NRT and backward digit recall emerged as significant predictors (Table 4).

A second regression analysis examined the contributions of these same measures at time 1 to syntax development. For this

Table 4 Standardized regression coefficients for predictors of change in vocabulary and grammar skills among boys with FXS over a 2-year period

\begin{tabular}{lcrr}
\hline & $\beta$ & $t$ & $p$ value \\
\hline Vocabulary change composite & & & \\
Leiter-R growth score & -0.02 & -0.15 & 0.88 \\
Autism symptom severity & 0.26 & 1.96 & 0.07 \\
Nonword repetition & $0.51^{*}$ & 3.67 & $<0.01$ \\
Backward digit recall & $0.56^{*}$ & 3.42 & $<0.01$ \\
Syntax change composite & & & \\
Leiter-R growth score & -0.24 & -1.08 & 0.29 \\
Autism symptom severity & -0.08 & -0.46 & 0.65 \\
Nonword repetition & $0.46^{*}$ & 2.47 & $<0.05$ \\
Backward digit recall & 0.45 & 2.03 & 0.06 \\
\hline
\end{tabular}

$* p<0.05$, statistically significant analysis, a syntax change composite was created by adding standardized change scores for TROG-2 and CASL-SC tests. For three participants for whom CASL-SC scores were not available at both time points, only the TROG-2 change score was used. Results of this regression analysis revealed that these predictors accounted for $40 \%$ of the variance in syntax development, $F(4,23)=3.20, p<0.01$. Nonword repetition was the only significant predictor of change in syntax (Table 4).

\section{Discussion}

The current study was designed to investigate the role of earlier phonological and verbal working memory skills in the acquisition of vocabulary and syntax in adolescents with FXS over a 2-year period. Preliminary analyses confirm two patterns derived from previous research on this population. First, girls with FXS tend to outperform boys across a range of language and memory tasks. Interestingly, girls in our sample not only exhibited more advanced language ability at the outset of the study, but they also demonstrated a significantly faster rate of acquisition of new vocabulary and syntax skills over the 2-year period. Second, language and memory scores at any given time point were closely associated with nonverbal cognitive ability. This suggests that, as with other populations, language skills have a strong relationship with general intellectual functioning.

In terms of the principal research questions, the present study extends findings from previous work by identifying how specific neuropsychological factors (phonological and working memory) are associated with growth in language during adolescence in FXS. In particular, results showed that among affected males, phonological memory is a significant predictor of acquisition of vocabulary and syntax over a period of 2 years. Further, phonological memory is more strongly related to language growth than nonverbal cognitive ability or autism symptom expression. One possible explanation for this finding is that, due to developmental delays among boys with FXS, the ability to maintain online phonological representations continues to be critical for language acquisition well into adolescence. Indeed, many of the males in our sample were functioning at the outset of our study at a level comparable to 4- to 6-year-old typically developing children with respect to vocabulary and syntax skill; this is an age during which phonological memory has strong relationships with vocabulary acquisition in typically developing children (Gathercole and Baddeley 1989). Baddeley et al. (1998) have argued that learning syntactic rules also depends on the ability to maintain multiword utterances in phonological memory. Given the relationship between nonword repetition skills and growth in syntax seen among boys with FXS, it 
seems likely that phonological memory skills place important constraints on acquisition of syntax during this time period as well. Interestingly, neuroimaging studies of males with FXS have shown a consistent pattern of reduced size of the cerebellar vermis, as well as increased size of the caudate nucleus (Lightbody and Reiss 2009); these structures and their connections with the frontal lobes are thought to be important correlates of phonological memory (Misciagna et al. 2010; Ben-Yehudah et al. 2007). Differences in the volume of these structures are also seen in females, but are more marked in those with lower levels of FMRP expression (Lightbody and Reiss 2009).

Another key finding of this study is that a verbal working memory task requiring active manipulation of items held in memory (i.e., backward digit span) accounted for unique variation in vocabulary development in boys with FXS. One explanation for this relationship is that boys with higher levels of cognitive control may be better able to attend to and organize representations in order to attach labels and meaning to objects. In addition, they may be better equipped to systematically access vocabulary knowledge to perform well on the type of standardized vocabulary tests administered in this study, as well as in a range of everyday speaking and listening tasks. Note that, although this verbal working memory measure did not significantly predict gains in syntax (when controlling for phonological memory), the relationship was in the predicted direction. Thus, studies using larger sample sizes may be needed to detect such a relationship.

Why were the relationships between memory measures and language growth seen in boys not observed for girls with FXS? One possible explanation is that our sample of affected girls may not have been large enough to detect these relationships. An important limitation of the current study is that the sample of girls is relatively small in comparison to boys. However, there was no trend in the data to indicate the presence of a stable relationship between phonological or working memory skills at time 1 and language growth among the girls. In fact, 7 of the 15 correlations were in the opposite direction than would be expected if there was a consistent relationship. Further, removing the covariates from the analyses also did not change the lack of relationship between the predictors and language outcomes. Thus, the data provide little evidence that a larger sample of girls would reveal an association between phonological and working memory and language development in the girls during the 2-year span.

A second explanation for the lack of relationship between memory measures and language growth might be that girls with FXS at this age demonstrate significantly more advanced language skills compared with boys. Indeed, mean scores for different language skills at time 1 among girls in our sample were roughly equivalent to normative data from 7 - to 10 -year-old children. This is beyond the age range during which strong associations have been found between phonological memory and vocabulary development in typically developing children (Gathercole et al. 1992). One might speculate that other factors such as educational programming or social context may, therefore, be more relevant predictors of language growth among adolescent girls with FXS. A number of studies have shown that reading habits among typically developing children have an increasing influence on vocabulary development during school age (Gathercole et al. 1992; Cunningham and Stanovich 1991). Thus, extent of exposure to print may be a better predictor of language growth for adolescent girls than working memory abilities. Note that this does not rule out the possibility that phonological and working memory skills are critical factors in earlier language development among affected girls. Given the current findings and the relative paucity of information about language development in girls with FXS in general (Murphy and Abbeduto 2003), this is an area in need of further research.

The association between phonological and verbal working memory and language development in boys with FXS suggests several potential strategies to improve or modify interventions for these individuals. A first step could be to increase awareness among educators and treatment providers of ways that processing limitations of students might impact their ability to comprehend spoken language as well as learn new language forms. For children with limited phonological and working memory, breaking down instructions into smaller parts, frequent repetition, and presentation of new language forms in varied contexts might increase the child's chance of learning success. External memory aids, such as pictures, diagrams, or lists may also be helpful. In a recent review paper aimed at practitioners, Gathercole and Alloway (2006) provide additional strategies for remedial support for individuals with working memory impairments. An important task for future research is to evaluate the efficacy of those strategies for FXS. A second strategy might be to directly train working memory processes. This could be accomplished with a variety of activities focused on enhancing rehearsal or working memory capacity that can be adapted based on the individual's ability and progress. Several programs available commercially or on the Internet are reviewed in an article by Montgomery et al. (2010). Whether these programs can be successful at enhancing working memory skills in FXS should be empirically tested. In any event, the results of the present study suggest that interventions focused only on increasing the intensity and structure of exposure to language may be limited in their effectiveness for adolescent boys with FXS without also considering the role of phonological and verbal working memory. Whether this can be best accomplished by use of compensatory strategies (such as providing visual supports 
along with language exposure) or by direct remediation of working memory is an important question for future research.

Although the present study is the first to identify cognitive factors contributing to language growth in adolescents with FXS, the study also has several limitations. As noted above, the sample size of female participants, while relatively large compared with many studies of FXS, may contribute to low statistical power to detect effects in this group. Further, additional research focused on girls with FXS at younger ages would reveal whether phonological and working memory abilities exert a more significant influence on language development during preschool or early school age for this group. It is possible that the age range of girls enrolled in the present study (adolescence) missed the developmental "window" for which these skills contribute critically to language growth. Furthermore, comparisons of individuals with FXS to other populations associated with language impairment and intellectual disability (e.g., Down syndrome, Williams syndrome) could more clearly delineate whether the cognitive risk factors identified in the current study play a role in language growth in boys with FXS due to syndrome-specific characteristics or whether these relationships primarily reflect a certain intellectual/developmental trajectory that is common across populations.

A second limitation of this research is that all of our measures of phonological and working memory relied on verbal output. Consequently, speech production/articulation difficulties may have contributed to task performance among participants with FXS. Future studies could include tasks that reduce or eliminate articulatory demands (e.g., Jarrold et al. 2002) in order to investigate the role of speech production difficulties in phonological and working memory performance in FXS. In addition, only a single measure of verbal working memory was obtained in the current study. The significant relationship of this measure to vocabulary growth in boys with FXS suggests that the degree of cognitive load and manipulation may be an important constraint in language learning in this population. However, future studies using additional measures of verbal working memory and cognitive control are needed to more fully elaborate the relationships suggested by our findings.

Finally, although we structured the testing situation to minimize the effects of attentional limitations and anxiety on task performance, these impairments are a central feature of the FXS phenotype and their impact cannot be completely eliminated. Indeed, in many natural language processing situations, attention and anxiety cannot be easily controlled and are likely to exert a powerful influence on performance. For example, girls with FXS who have higher cognitive capacities may be particularly aware that their performance is being evaluated in a testing situation; this could potentially result in some anxiety affecting performance on novel or attention-demanding tasks such as working memory tests. An important task for future research, therefore, is to expand the current set of predictors in the model to include other phenotype-relevant predictors such as attention and anxiety. More generally, FXS is associated with a complex, multidimensional phenotype. The current study has taken an important first step to identify specific cognitive factors that contribute to language growth, but there are undoubtedly other cognitive, motor, social-emotional, and developmental variables that contribute to language development that have not yet been identified and are worthy of future investigation. Additionally, the current study does not have data to contribute regarding the ways in which education, family, and other environmental factors interact with phonological and working memory skills to shape language development in FXS. Thus, future research in this area should address both additional complexities within the FXS phenotype as well as these environmental sources of variation.

\section{Conclusions}

Studies of cognitive mechanisms that support the development of language skill have the potential to improve the way language disorders are identified and treated in individuals with neurodevelopmental disabilities. In this study, we demonstrated that two cognitive skills, phonological and verbal working memory, predicted growth in language skills among adolescent boys with FXS but not among girls, even after controlling for nonverbal cognitive ability and severity of autism symptoms. These results challenge the view that gender differences in language outcomes can be fully explained by differences in overall intellectual functioning. Future studies focused on additional mechanisms affecting language change, including environmental factors, could provide additional insight into the pathogenesis of language impairments in this population and suggest further avenues for treatment.

Acknowledgements This work was supported by National Institutes of Health grant R01 HD024356 awarded to L. Abbeduto and P30 HD003352 awarded to the Waisman Center, University of Wisconsin Madison. Special thanks to Susen Schroeder for the assistance with establishing task reliability. We also greatly appreciate the contributions of the children and families who participated in this research.

\section{References}

Abbeduto L, Murphy MM, Cawthon SW, Richmond EK, Weissman MD, Karadottir S, et al. Receptive language skills of adolescents and young adults with Down syndrome or fragile X syndrome. Am J Ment Retard. 2003;108(3):149-60.

Abbeduto L, Brady N, Kover ST. Language development and fragile $\mathrm{X}$ syndrome: profiles, syndrome-specificity, and withinsyndrome differences. Ment Retard Dev Disabil Res Rev. 2007;13(1):36-46. 
Acheson DJ, MacDonald MC. Verbal working memory and language production: common approaches to the serial ordering of verbal information. Psychol Bull. 2009;135(1):50-68.

Alloway TP, Gathercole SE, Pickering SJ. Verbal and visuospatial short-term and working memory in children: are they separable? Child Dev. 2006;77(6):1698-716.

Archibald LMD, Gathercole SE. Short-term and working memory in specific language impairment. Int $\mathrm{J}$ Lang Commun Disord. 2006;41(6):675-93

Awh E, Jonides J, Smith EE, Schumacher EH, Koeppe RA, Ktaz S. Dissociation of storage and rehearsal in verbal working memory: evidence from positron emission tomography. Psychol Sci. 1996;7(1):25-31.

Baddeley AD. Working memory. New York: Oxford University Press; 1986.

Baddeley AD. Working memory and language: an overview. J Commun Disord. 2003;36(3):189-206.

Baddeley AD, Gathercole S, Papagno C. The phonological loop as a language learning device. Psychol Rev. 1998;105(1):158-73.

Baker S, Hooper S, Skinner M, Hatton D, Schaaf J, Ornstein P, et al. Working memory subsystems and task complexity in young boys with fragile X syndrome. J Intellect Disabil Res. 2011;55(1):19-29.

Belser RC, Sudhalter V. Conversational characteristics of children with fragile X syndrome: repetitive speech. Am J Ment Retard. 2001;106(1):28-38.

Ben-Yehudah G, Guediche S, Fiez JA. Cerebellar contributions to verbal working memory: beyond cognitive theory. Cerebellum. 2007;6(3):193-201.

Bishop DVM. Test for Reception of Grammar, Version 2. London: The Psychological Corporation; 2003.

Bowey J. Nonword repetition and young children's receptive vocabulary: a longitudinal study. Appl Psycholinguist. 2001;22(3):441-69.

Brock J, Jarrold C. Language influences on verbal short-term memory performance in Down syndrome: item and order recognition. $\mathrm{J}$ Speech Lang Hear Res. 2004;47(6):1334-46.

Cairns P, Jarrold C. Exploring the correlates of impaired non-word repetition in Down syndrome. Br J Dev Psychol. 2005;23:401-16.

Carrow-Woolfolk E. Comprehensive Assessment of Spoken Language. Circle Pines: American Guidance Service; 1999.

Chapman RS. Language learning in Down syndrome: the speech and language profile compared to adolescents with cognitive impairment of unknown origin. Down Syndrome Res Pract. 2006;10 (2):61-6.

Cohen J. A coefficient of agreement for nominal scales. Educ Psychol Meas. 1960;20:37-46.

Conklin HM, Curtis CE, Katsanis J, Iacono WG. Verbal working memory impairment in schizophrenia patients and their firstdegree relatives: evidence from the digit span task. Am J Psychiatry. 2000;157(2):275-7.

Cornish KM, Munir F, Cross G. Differential impact of the FMR-1 full mutation on memory and attention functioning: a neuropsychological perspective. J Cogn Neurosci. 2001;13(1):144-50.

Cornish KM, Kogan CS, Li L, Turk J, Jacquemont S, Hagerman RJ. Lifespan changes in working memory in fragile $\mathrm{X}$ premutation males. Brain Cogn. 2009;69(3):551-8.

Cowan N. Attention and memory: an integrated framework. Oxford, England: Oxford University Press 1995.

Cronister A, Hagerman RJ, Wittenberger M, Amiri K. Mental impairment in cytogenetically positive fragile $\mathrm{X}$ females. Am J Med Genet. 1991;38(2-3):503-4.

Cunningham AE, Stanovich KE. Tracking the unique effects of print exposure in children: associations with vocabulary, general knowledge, and spelling. J Educ Psychol. 1991;83(2):264-74.

D'Esposito M. Maintenance versus manipulation of information held in working memory: an event-related fMRI study. Brain Cogn. 1999;41(1):66-86.
D'Esposito M, Aguirre GK, Zarahn E, Ballard D, Shin RK, Lease J. Functional MRI studies of spatial and nonspatial working memory. Cog Brain Res. 1998;7(1):1-13.

de Vries BB, Wiegers AM, Smits AP, Mohkamsing S, Duivenvoorden HJ, Fryns JP, et al. Mental status of females with an FMR1 gene full mutation. Am J Hum Genet. 1996;58(5):1025-32.

Demark JL, Feldman MA, Holden JJ. Behavioral relationship between autism and fragile X syndrome. Am J Ment Retard. 2003;108 (5):314-26.

Dollaghan CA, Campbell TF. Nonword repetition and child language impairment. J Speech Lang Hear Res. 1998;41:1136-46.

Dunn LM, Dunn LM. Peabody Picture Vocabulary Test. 3rd ed. Circle Pines: American Guidance Service; 1997.

Durkin K, Simkin Z, Knox E, Conti-Ramsden G. Specific language impairment and school outcomes. II: educational context, student satisfaction, and post-compulsory progress. Int J Lang Commun Disord. 2009;44(1):36-55.

Ellis Weismer S, Tomblin JB, Zhang X, Buckwalter P, Chynoweth JG, Jones $\mathrm{M}$. Nonword repetition performance in school-age children with and without language impairment. J Speech Lang Hear Res. 2000;43(4):865-78.

Fernandez-Carvajal I, Walichiewicz P, Xiaosen X, Pan R, Hagerman PJ, Tassone F. Screening for expanded alleles of the FMR1 gene in blood spots from newborn males in a Spanish population. J Mol Diagn. 2009;11(4):324-9.

Finestack LH, Richmond EK, Abbeduto L. Language development in individuals with fragile X syndrome. Top Lang Disord. 2009;29 (2):133-48.

Gathercole SE, Alloway TP. Practitioner review: short-term and working memory impairments in neurodevelopmental disorders: diagnosis and remedial support. J Child Psychol Psychiatry. 2006;47(1):4-15.

Gathercole SE, Baddeley AD. Evaluation of the role of phonological STM in the development of vocabulary in children: a longitudinal study. J Mem Lang. 1989;28(2):200-13.

Gathercole SE, Baddeley AD. Phonological memory deficits in language disordered children: is there a causal connection? J Mem Lang. 1990;29(3):336-60.

Gathercole SE, Willis C, Emslie H, Baddeley AD. Phonological memory and vocabulary development during the early school years: a longitudinal study. Dev Psychol. 1992;28(5):887-98.

Gotham K, Pickles A, Lord C. Standardizing ADOS scores for a measure of severity in autism spectrum disorders. J Autism Dev Disord. 2009;39(5):693-705.

Graf Estes K, Evans JL, Else-Quest NM. Differences in the nonword repetition performance of children with and without specific language impairment: a meta-analysis. J Speech Lang Hear Res. 2007;50(1):177-95.

Grant J, Karmiloff-Smith A, Gathercole SE, Paterson S, Howlin P, Davies $\mathrm{M}$, et al. Phonological short-term memory and its relationship to language in Williams syndrome. Cogn Neuropsychiatr. 1997;2(2):81-99.

Hagerman PJ. The fragile X prevalence paradox. J Med Genet. 2008;45(8):498-9.

Hall SS, Lightbody AA, Hirt M, Rezvani A, Reiss AL. Autism in fragile X syndrome: a category mistake? J Am Acad Child Adolesc Psychiatry. 2010;49(9):921-33.

Harris SW, Hessl D, Goodlin-Jones B, Ferranti J, Bacalman S, Barbato I, et al. Autism profiles of males with fragile $\mathrm{X}$ syndrome. Am J Ment Retard. 2008;113(6):427-38.

Hessl D, Nguyen DV, Green C, Chavez A, Tassone F, Hagerman RJ, et al. A solution to limitations of cognitive testing in children with intellectual disabilities: the case of fragile $\mathrm{X}$ syndrome. $\mathrm{J}$ Neurodev Disord. 2009;1(1):33-45.

Holm S. A simple sequentially rejective multiple test procedure. Scand J Stat. 1979;6:65-70. 
Hooper SR, Hatton DD, Baranek GT, Roberts JP, Bailey DB. Nonverbal assessment of IQ, attention, and memory abilities in children with fragile-X syndrome using the Leiter-R. J Psychoeduc Assess. 2000;18(3):255-67.

Hooper SR, Hatton D, Sideris J, Sullivan K, Hammer J, Schaaf J, et al. Executive functions in young males fragile $\mathrm{X}$ syndrome in comparison to mental age-matched with controls: baseline findings from a longitudinal study. Neuropsychology. 2008;22(1):36-47.

Jarrold C, Baddeley AD, Phillips CE. Verbal short-term memory in Down syndrome: a problem of memory, audition, or speech? J Speech Lang Hear Res. 2002;45(3):531-44.

Kaufmann WE, Cortell R, Kau AS, Bukelis I, Tierney E, Gray RM, et al. Autism spectrum disorder in fragile $\mathrm{X}$ syndrome: communication, social interaction, and specific behaviors. Am J Med Genet A. 2004;129A(3):225-34.

Kover ST, Abbeduto L. Expressive language in male adolescents with fragile X syndrome with and without comorbid autism. J Intellect Disabil Res. 2010;54(3):246-65.

Kuschner ES, Bennetto L, Yost K. Patterns of nonverbal cognitive functioning in young children with autism spectrum disorders. J Autism Dev Disord. 2007;37(5):795-807.

Lanfranchi S, Cornoldi C, Drigo S, Vianello R. Working memory in individuals with fragile X syndrome. Child Neuropsychol. 2009;15 (2):105-19.

Laws G. The use of nonword repetition as a test of phonological memory in children with Down syndrome. J Child Psychol Psychiatry. 1998;39(8):1119-30.

Laws G, Gunn D. Phonological memory as a predictor of language comprehension in Down syndrome: a five-year follow-up study. J Child Psychol Psychiatry. 2004;45(2):326-37.

Lewis P, Abbeduto L, Murphy M, Richmond E, Giles N, Bruno L, et al. Cognitive, language and social-cognitive skills of individuals with fragile $\mathrm{X}$ syndrome with and without autism. J Intellect Disabil Res. 2006;50:532-45.

Lightbody AA, Reiss AL. Gene, brain, and behavior relationships in fragile X syndrome: evidence from neuroimaging studies. Dev Disabil Res Rev. 2009;15(4):343-52.

Loesch DZ, Huggins RM, Hagerman RJ. Phenotypic variation and FMRP levels in fragile X. Ment Retard Dev Disabil Res Rev. 2004;10(1):31-41.

Logie RH. Visuo-spatial working memory. Hillsdale: Erlbaum; 1995.

Lord C, Rutter M, Le Couteur A. Autism Diagnostic InterviewRevised: a revised version of a diagnostic interview for caregivers of individuals with possible pervasive developmental disorders. J Autism Dev Disord. 1994;24(5):659-85.

Lord C, Rutter M, DiLavore P, Risi S. Autism Diagnostic Observation Schedule. Los Angeles: Western Psychological Services; 1999.

Lycke C, Specht K, Ersland L, Hugdahl K. An fMRI study of phonological and spatial working memory using identical stimuli. Scand J Psychol. 2008;49(5):393-401.

MacDonald M, Christianson M. Reassessing working memory: a reply to Just and Carpenter (1992) and Waters and Caplan (1996). Psychol Rev. 2002;109:35-54.

Mazzocco MM. Advances in research on the fragile X syndrome. Ment Retard Dev Disabil Res Rev. 2000;6(2):96-106.

Mazzocco MM, Thompson L, Sudhalter V, Belser RC, Lesniak-Karpiak $\mathrm{K}$, Ross JL. Language use in females with fragile $\mathrm{X}$ or Turner syndrome during brief initial social interactions. J Dev Behav Pediatr. 2006;27(4):319-28.

McDuffie A, Abbeduto L, Lewis P, Kover S, Kim JS, Weber A, et al. Autism spectrum disorder in children and adolescents with fragile $\mathrm{X}$ syndrome: within-syndrome differences and age-related changes. Am J Intellect Dev Disabil. 2010;115(4):307-26.

McDuffie A, Kover ST, Abbeduto L, Lewis P, Brown T. Profiles of receptive and expressive language in males with comorbid fragile x syndrome and autism. Am J Intellect Dev Disabil. in press.
Mervis CB. Cross-etiology comparisons of cognitive and language development. In: Rice ML, Warren SF, editors. Developmental language disorders: from phenotypes to etiologies. Mahwah: Lawrence Erlbaum Associates; 2004. p. 153-85.

Mervis CB, Klein-Tasman BP. Methodological issues in groupmatching designs: $\alpha$ levels for control variable comparisons and measurement characteristics of control and target variables. J Autism Dev Disord. 2004;34(1):7-17.

Misciagna S, Iuvone L, Mariotti P, Silveri MC. Verbal short-term memory and cerebellum: evidence from a patient with congenital cerebellar vermis hypoplasia. Neurocase. 2010;16 (2): 119-24.

Montgomery JW, Magimairaj BM, Finney MC. Working memory and specific language impairment: an update on the relation and perspectives on assessment and treatment. Am J Speech Lang Pathol. 2010;19:78-94

Munir F, Cornish KM, Wilding J. Nature of the working memory deficit in fragile-X syndrome. Brain Cogn. 2000;44(3):387-401.

Murphy MM, Abbeduto L. Language and communication in fragile $\mathrm{X}$ syndrome. Int Rev Res Ment Retard. 2003;27:83-119.

Murphy MM, Abbeduto L. Gender differences in repetitive language in fragile $\mathrm{X}$ syndrome. $\mathrm{J}$ Intellect Disabil Res. 2007;51:387-400.

Paulesu E, Frith CD, Frackowiak RSJ. The neural correlates of the verbal component of working memory. Nature. 1993;362:342-5.

Pesso R, Berkenstadt M, Cuckle H, Gak E, Peleg L, Frydman M, et al. Screening for fragile $\mathrm{X}$ syndrome in women of reproductive age. Prenat Diagn. 2000;20(8):611-4.

Philofsky A, Hepburn S, Hayes A, Hagerman R, Rogers SJ. Linguistic and cognitive functioning and autism symptoms in young children with fragile X syndrome. Am J Ment Retard. 2004;109 (3):208-18.

Pickering S, Gathercole S. The Working Memory Test Battery for Children. London: The Psychological Corporation; 2001.

Roberts J, Martin GE, Moskowitz L, Harris AA, Foreman J, Nelson L. Discourse skills of boys with fragile $\mathrm{X}$ syndrome in comparison to boys with Down syndrome. J Speech Lang Hear Res. 2007;50 (2):475-92.

Robinson BF, Mervis CB, Robinson BW. The roles of verbal shortterm memory and working memory in the acquisition of grammar by children with Williams syndrome. Dev Neuropsychol. 2003;23(1-2):13-31.

Roid G, Miller L. Leiter International Performance Scale-Revised. Wood Dale: Stoelting; 1997.

Skinner M, Hooper S, Hatton DD, Robert J, Mirrett P, Schaaf J, et al. Mapping nonverbal IQ in young boys with fragile $\mathrm{X}$ syndrome. Am J Med Genet A. 2005;132A(1):25-32.

Smith EE, Jonides J. Storage and executive processes in the frontal lobes. Science. 1999;283(5408):1657-61.

Smith EE, Jonides J, Koeppe RA. Dissociating verbal and spatial working memory using PET. Cereb Cortex. 1996;6(1):11-20.

Sudhalter V, Scarborough HS, Cohen IL. Syntactic delay and pragmatic deviance in the language of fragile $\mathrm{X}$ males. Am J Med Genet. 1991;38(2-3):493-7.

Van Agt H, Verhoeven L, Van Den Brink G, De Koning H. The impact on socio-emotional development and quality of life of language impairment in 8-year-old children. Dev Med Child Neurol. 2011;53(1):81-8.

Wager TD, Smith EE. Neuroimaging studies of working memory: a metaanalysis. Cogn Affect Behav Neurosci. 2003;3(4):255-74.

Walter H, Bretschneider V, Gron G, Zurowski B, Wunderlich AP, Tomczak R, et al. Evidence for quantitative domain dominance for verbal and spatial working memory in frontal and parietal cortex. Cortex. 2003;39(4-5):897-911.

Williams K. Expressive Vocabulary Test. Circle Pines: American Guidance Service; 1997. 